\title{
Reverse Time Migration in Viscoacoustic Media
}

Adriano de Souza Junior, ${ }^{1}$ Felipe Timóteo da Costa ${ }^{1}$, Marco Antonio Cetale Santos ${ }^{1}$ 1 - GISIS - UFF

Copyright 2019, SBGf - Sociedade Brasileira de Geofísica

This paper was prepared for presentation during the $16^{\text {th }}$ International Congress of the Brazilian Geophysical Society held in Rio de Janeiro, Brazil, 19-22 August 2019.

Contents of this paper were reviewed by the Technical Committee of the $16^{\text {th }}$ International Congress of the Brazilian Geophysical Society and do not necessarily represent any position of the SBGf, its officers or members. Electronic reproduction or the witten consent of the Brazilian Geophysical Society is prohibited.

Abstract

Seismic Migration has become one of the most important steps of seismic data processing, mainly with the necessity no increase resolution of deep structures, specially the ones under carbonates and salt, that cause a big atenuation on seismic waves, making it difficult to produce images with true amplitudes for AVO studies and $4 D$ (time lapse) seismic data. With this being said, this work shows a preliminary mehodology for implementing a viscoscalar wave equation and viscoacoustic migration to solve this problems.

\section{Introduction}

Much is being done on seismic processing and imaging since computation allowed geoscientists to run larger programs. In this path, Reverse time migration (RTM) has diferentiated itself from the other imaging techniques, because of its capability to image high deep structures, since it uses the two-way wave equation and it is not ray based. Despite that, the image generated commonly has low resolution and its amplitude is relative. In this way, many viscoacoustic wave equations have been developed, as pointed by Yang et al(2014). This work aims to present a simple implementation for a viscoacoustic RTM, that has the same core and also generates a true amplitude image.

\section{Method}

\section{Viscoacoustic wave Equation}

Seismic Modelling has an important role for many techniques in the seismic processing, and is the core of Reverse Time migration (RTM). Despite that, one of the main problems is to produce data with a correct amplitude due to absortion. To solve this problem, one common processing flow is to, after the seismic data is prepared to be migrated, apply a $\mathrm{Q}$ correction for the phase, migrate the data with an acoustic operator and apply a Q correction for the amplitude. For this work, we used the viscoscalar wave equation proposed by Deng et al(2007), a simple approach for solving, more accurately, the amplitude problem once the phase is already corrected, because, as explained by Yang et al (2014), since it is not frequency dependent and is not based on the constant $\mathrm{Q}$ model, the frequency peak does not change with the attenuation, hence, it is not suited for $\mathrm{Q}$ estimation using the slope of the spectral ratio and can not represent frequency-dependent dispersion. The viscoscalar equation is described as:

$$
\frac{p s i g}{v} \frac{\partial^{2} P}{\partial t}+\frac{1}{v^{2}} \frac{\partial^{2} P}{\partial t^{2}}=\frac{\partial^{2} P}{\partial x^{2}}+\frac{\partial^{2} P}{\partial z^{2}}
$$

Were $P$ is the pressure field, $x$ and $z$ are the space horizontal and vertical components, $t$ is the time variable, $v$ is the velocity, $g$ is the absorption coefficient, described by:

$$
g=\frac{2 \pi f}{v Q}
$$

Where $f$ is the reference frequency, that we approximated to the wavelet central frequency. Q is the quality factor. For small Q values, the energy is fastly damped. Psi is the parameter that controls which wave equation we are modelling: if $\mathrm{Psi}=0$, we have an acoustic wave equation; if it is equal to 1 , a forward absortive wave equation were energy is damped and, if it is equal to -1 , a reverse propagation, with the amplitudes enhanced.

\section{Seismic Modeling}

In order to use it for computational wave propagation, we discretized it using the finite differences method, on a simple regular grid, approximating the derivatives by the Taylor Series expansion to the fourth order.

Also, the problem is treated with initial condition of the pressure fields equal to zero. We used a $30 \mathrm{~Hz}$ Ricker Wavelet simulating the source and receivers on the top of the model. The border problem, since we are representing a virtually infinite media physical experiment in a limited environment with borders, were solved using a common approach, i.e, Cerjan damping layers, that smoothly reduces the wave amplitude from the model to the border, and Reynolds non-reflexive wave equation at each border. Q models were approximated using Li(1993) empirical equation to convert compressional velocity in Quality factor:

$$
Q=3.516 \times\left(v p^{2,2}\right) \times\left(10^{-6}\right)
$$




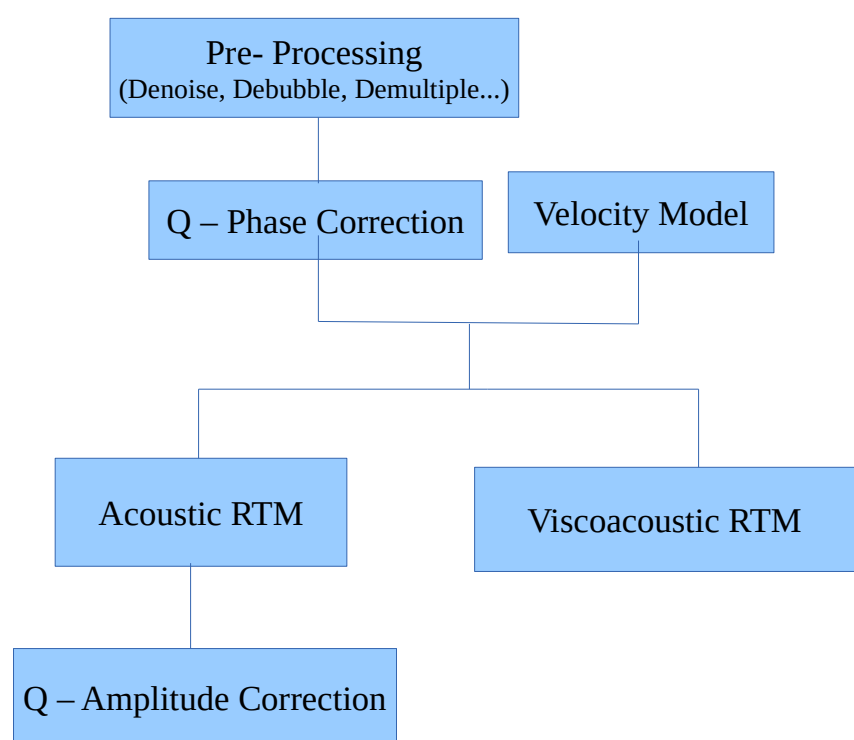

Figure 1. Seismic Processing sugested flows with RTM. On the left branch, a default processing flow with acoustic migration and a $\mathrm{Q}$ correction on the stack. On the right, a Viscoacoustic migration correcting the amplitude on the process.

\section{Migration}

To construct the image, RTM migration were performed. As usual, the source wave field (Downgoing) and the Receiver wave field (Upgoing) were reconstructed using the same modeling operator and borders to generate the seismograms. The only differences were: the velocity model, that in order to avoid reflections it was smoothed both vertically and laterally using a Gaussian filter; the wave equation used, depending if we want to migrate the data using acoustic or viscoacoustic operator. With the wave fields reconstructed, an image condition must be used to match them. The one used was source normalized cross-correlation (Chattopadhyay and Mcmechan, 2008) were we multiply both Upgoing and Downgoing wave fields at each time step, divide them by the auto-correlation of the Downgoing wave field and add the result to the shot image. After generating an image for each shot, they were stacked and a laplacian operator were applied in order to remove the low-frequency noise that is generated in the modeling/migration process, as suggested by Zhang et al(2009).

\section{Results}

Some tests were made on the Marmousi model. Viscoacoustic forward modeling was used to generate the synthetic seismograms and an Acoustic and Viscoacoustic RTM are compared.
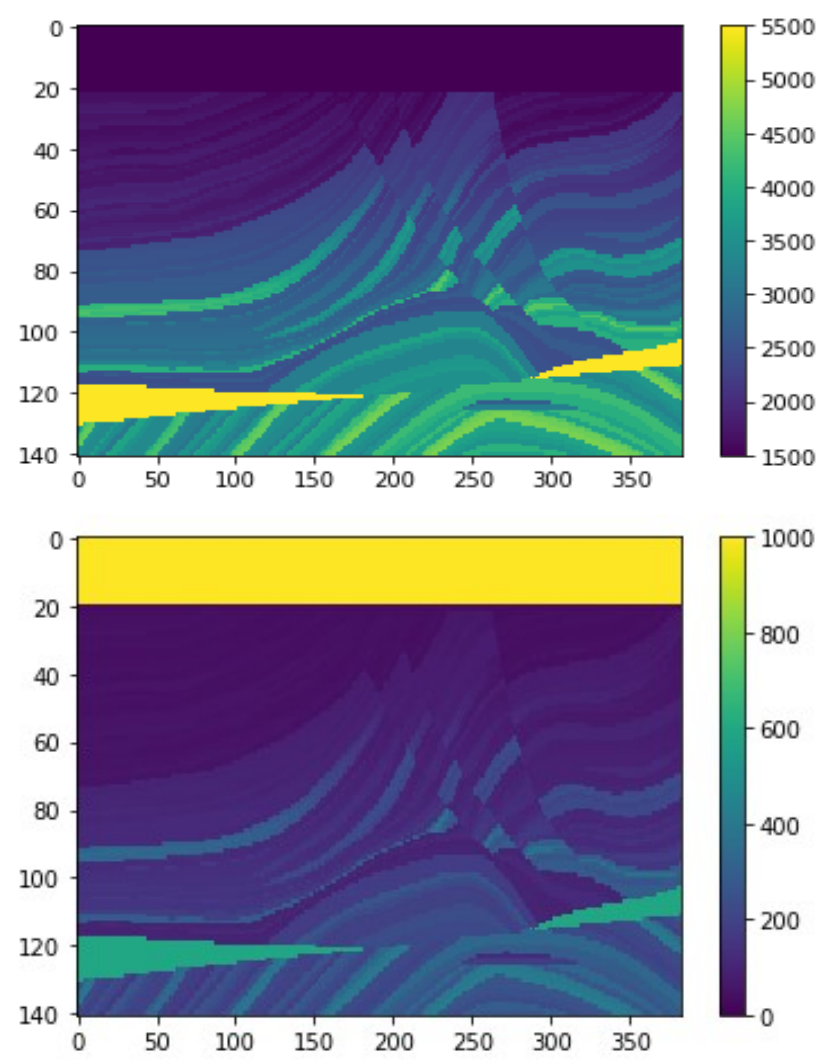

Figure 2. Marmousi Velocity model(top) and Q model(bottom) used for the modeling and migration. Q factor of water was extrapolated to a very high value in order to simulate zero attenuation.

As we can see, the image resolution of the viscoacoustic RTM is better just changing the wave equation for the migration, meaning that no big changes are necessary in the used seismic processing flow. Also, we generate seismic data with correct amplitude values if we input the correct Q factor model, in order not to show stronger or weaker amplitudes than they should be. 

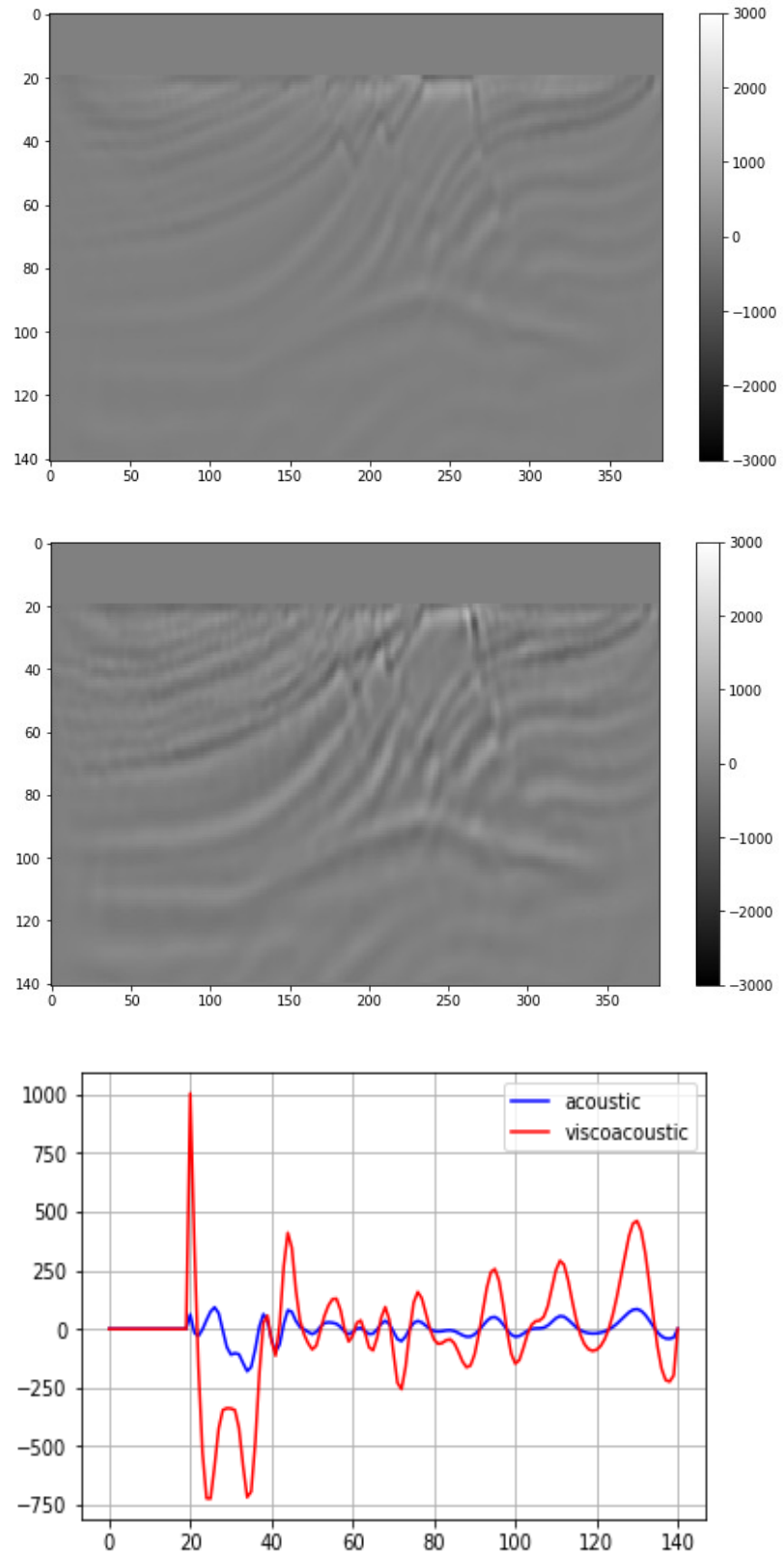

Figure 3. Comparison between acoustic and viscoacoustic reverse time migration over viscoacoustic seismograms. The top one is the acoustic and the midle is the viscoacoustic. As expected, the acoustic migration has weaker amplitudes. In the bottom, a seismic trace showing the amplitudes difference. Low frequency noises are also amplificated.

\section{Conclusions}

A Viscoacoustic RTM were presented using Deng(2007) viscoscalar wave equation, that is able to migrate data with its correct amplitude once the phase has been corrected. Also, a Gaussian filter is used to mitigate the effects of the low amplitude noise generated by the source normalized cross-correlation imaging condition. Comparing the benchmark seismic data generated to the Viscoacoustic-RTM, the Acoustic and the Q-corrected Acoustic, we are able to see that seismic amplitude, frequency content and resolution increase considerably, without big changes on the processing flow, making it easier to implement, since the RTM methodology is the same. However, the computational cost increases, meaning that, depending on the objective, one should think about using it or apply the one directional inverse $\mathrm{Q}$ filter, exchanging time to a more accurate seismic amplitude.

This is a preliminary result of an undergraduation research and has future steps. A more robust benchmark will be designed to test if the viscoacoustic operator is well implemented and, other wave equations will be tested, as well as their gains and limitations when compared to the presented in this work.

\section{Acknowledgments}

We would like to thank GISIS for providing the necessary tools to perform this work. Also, the first author would like to thank CNPq for the finantial support and JetBrains for providing the IDE where it was programmed.

\section{References}

Deng, F., and G. A. McMechan, 2008, Viscoelastic trueamplitude prestack reverse-time depth migration: Geophysics, 73, no. 4, S143-S155.

Zhu, Tieyuan \& Harris, Jerry \& Biondi, Biondo. (2014). QCompensated Reverse-Time Migration. Geophysics. 79. 10.1190/geo2013-0344.1.

Yang, Zongqing \& Liu, Yang \& Ren, Zhiming. (2014). Comparisons of visco-acoustic wave equations. Journal of Geophysics and Engineering. 11.025004. 10.1088/1742-2132/11/2/025004.

Li, Q.Z., 1993, High-Resolution seismic data processing: Petroleum Industry Press, China, 37-38

Chattopadhyay, Sandip \& Mcmechan, George. (2008). Imaging conditions for prestack reverse-time migration. Geophysics. 73. 10.1190/1.2903822.

Zhang, Yu \& Houston, Cggveritas \& Sun, James \& , Cggveritas \&, Singapore. (2019). Practical issues of reverse time migration: true-amplitude gathers, noise removal and harmonic-source encoding. 